\title{
Advances in Surgical Options for Medically Refractory Epilepsy
}

\author{
Paul R Gigante1 and Robert R Goodman²
}

\begin{abstract}
1. Resident, Department of Neurological Surgery, Columbia University Medical Center; 2. Chairman, Department of Neurosurgery, St Luke's-Roosevelt Hospital Center and Associate Professor of Clinical Neurological Surgery, Department of Neurological Surgery,
\end{abstract} The Neurological Institute of New York, Columbia University Medical Center, New York, US

\begin{abstract}
The evolution of surgical treatment for medically refractory epilepsy (MRE) has been influenced over the last decade by substantial advancements in imaging- and device-related technology, as well as an expanding assemblage of prospective data that support the utilisation of surgery for MRE. These data, which have grown to include randomised trials and long-term follow up for established surgery, as well as large series for investigational procedures, have demonstrated safe, efficacious results with proper patient selection. Prospective randomised trials of three surgically implanted neuromodulatory devices, vagus nerve stimulators, deep brain stimulators and responsive neurostimulators have demonstrated safety and significant seizure frequency reduction. Numerous studies have provided strong evidence for the efficacy and safety of temporal lobe resective surgery and recent studies have focused on applying alternative approaches to open resective surgery for patients presumed to have a medial temporal seizure focus. These alternatives include stereotactic radiosurgery, radiofrequency ablation and a magnetic resonance imaging (MRI)-guided laser technique for thermal ablation. Current evidence for these new surgical options for the treatment of medically refractory epilepsy will be presented and discussed.
\end{abstract}

\section{Keywords}

Epilepsy, deep brain stimulation, surgery, neurostimulation, neurostimulator, neuromodulation, refractory, radiosurgery, vagus, temporal lobe epilepsy, responsive

Disclosure: Paul R Gigante has no conflicts of interest to declare. Robert R Goodman serves as a consultant for NeuroPace, Inc. Received: 6 May 2012 Accepted: 8 June 2012 Citation: European Neurological Review, 2012;7(2):140-4 DOI:10.17925/ENR.2012.07.02.140 Correspondence: Paul R Gigante, Department of Neurological Surgery, Columbia University Medical Center, 710 West 168th street, 4th floor, New York, NY 10032, US. E: pg2223@columbia.edu

\section{Neuromodulation Devices Vagus Nerve Stimulation}

Vagus nerve stimulation (VNS), first used for seizure treatment in the 1880s, was approved by the US Food and Drug Administration (FDA) in 1997 after decades of animal studies demonstrating reduction of chemically induced seizures, ${ }^{1,2}$ and subsequent promising human trials beginning in the early 1990s. Since FDA approval, VNS technology has been improved, with smaller neurostimulator/battery and simplified wire and connection. After exposure of the left vagus nerve distal to the recurrent laryngeal nerve, two bipolar electrodes are placed around the nerve and connected to a subcutaneously implanted, programmable stimulation device below the level of the clavicle. Stimulation is typically at high frequency and cycles between periods on (typically 30 seconds) and off (typically several minutes). To date, the physiological mechanism of VNS on seizure activity remains incompletely understood. As identified broadly in neuronal networks involved in seizure pathophysiology, VNS studies indicate that stimulation influences activity in the thalamus and limbic structures, alters cerebral blood flow and influences neurotransmitter and amino acid concentrations. ${ }^{3-5}$

Initially, two blinded, randomised controlled trials comparing high and low VNS amplitude stimulation in patients over 12 years old with partial seizures demonstrated a significantly greater reduction in seizure frequency in the high-stimulation (25-28 \%) group compared to the low-stimulation (6-15\%) group. ${ }^{6,7}$ Multiple prospective and retrospective series followed, reporting seizure reduction outcomes in variable epilepsy populations.

Recently, the first meta-analysis of VNS trials identified 74 clinical studies containing outcomes data, of which 15 studies produced Class I, II, or III evidence. In a pooled analysis of 2,634 patients, the authors determined the efficacy of VNS to be a $\geq 50 \%$ reduction in seizure frequency in $50.6 \%$ of patients; a $\geq 90 \%$ seizure reduction in $12.2 \%$; and seizure freedom in $4.6 \%$ of patients. The mean seizure frequency reduction was $44.6 \%$ amongst 1,789 patients with available percentage reduction data. Despite a large volume of pooled data, the wide variability in follow-up, ranging from three months to five years, and non-controlled variables such as medication changes, indicate the continued need for a randomised controlled trial with long-term follow-up.

In an evaluation of predictors of response to VNS therapy, the authors determined a small but statistically significant trend toward a greater benefit in paediatric patients ( $<18$ years old) compared with adults ( $\geq 18$ years old). ${ }^{8}$ Identifying the efficacy of VNS in paediatric populations is particularly important because FDA approval in 1997 was for adults and adolescents $>12$ years old, based on trial data that 
was available at the time. Also notable from the meta-analysis was that children younger than six years old appeared to have a more significant decrease in seizure frequency $(62 \%)$ than older populations. The authors stratified outcomes by epilepsy aetiology where reported, though these data were limited to a significantly smaller pooled population (517 patients); the greatest benefit was found in patients with post-traumatic epilepsy and tuberous sclerosis

\section{Deep Brain Stimulation}

Deep brain stimulation (DBS) has proven efficacious in treating advanced Parkinson's disease via implantation in the subthalamic nucleus and globus pallidus interna, 90 and is currently under investigation for use in a number of other central nervous system (CNS) disorders such as depression, ${ }^{11}$ obsessive-compulsive disorder, ${ }^{12}$ Tourette's syndrome, ${ }^{13}$ and epilepsy. DBS surgery involves advancing a macroelectrode through the brain such that the cranial electrode tip terminates in a precise anatomic location, typically selected using a fine-cut pre-operative magnetic resonance image (MRI) in conjunction with stereotactic head-frame guidance. The tip of the electrode contains multiple electrical contacts, the settings of which can be adjusted on an outpatient basis using a subcutaneously implanted generator. Stimulation is programmed by the treating physician and is typically continuous. The generator is typically placed below the clavicle and connected to the cranial electrode via an extension wire, which can be performed as a separately staged procedure, or on the same day as the cranial electrode implantation.

Though DBS has been studied for treatment of refractory epilepsy in multiple anatomic targets since the 1980s - including the centromedian nucleus of the thalamus and the cerebellum - the most robust data have come from stimulation of the anterior nucleus of the thalamus (ANT) $)^{14,15}$ and the medial temporal lobe. ${ }^{16}$ These data led to the initiation of the Stimulation of the anterior nucleus of the thalamus for epilepsy (SANTE) trial, a multicentre, double-blinded, randomised trial of bilateral ANT stimulation for patients with partial seizures refractory to at least three anti-epileptic drugs (AEDS). Results of the trial were published in 2010, demonstrating a mean seizure frequency reduction during the three-month double-blinded phase of $36.3 \%$ in the 'on' group versus $12.1 \%$ in the 'off' group ( $p=0.041)$. During months four to 13 of the study, all patients were treated with unblinded stimulation 'on,' and afterward entered the long-term open-label period, during which stimulation parameters could vary freely; results after the blinded period demonstrated a seizure reduction of $41 \%$ at 13 months and $56 \%$ at 25 months, with only two patients achieving seizure freedom from months four to $13 .{ }^{17}$ Though DBS received Conformité Européenne (CE) Mark approval for medically refractory epilepsy (MRE) in 2010, the FDA is currently awaiting further studies for consideration of DBS approval for MRE. ${ }^{18}$

To date, the efficacy of medial temporal lobe programmed stimulation has been limited to small-scale studies demonstrating a modest benefit. Two registered clinical trials are ongoing to investigate the efficacy of bilateral hippocampal stimulation. The first, named Controlled randomized stimulation versus resection (CoRastiRis), designed to randomise adult patients with medically refractory partial seizures to three treatment arms: medial temporal lobe resection, immediate hippocampal neurostimulation, or implanted electrode with delayed stimulation. ${ }^{19}$ The second, the Multicenter study of hippocampal electrical stimulation (METTLE), was designed to randomise adult patients with medically refractory epilepsy to hippocampal electrode implantation with stimulation or hippocampal electrode implantation without stimulation..$^{20}$

\section{Responsive Neurostimulation}

One of the newest surgically implantable devices employed for the treatment of MRE is the Responsive NeurostimulatorTM (RNS ${ }^{\mathrm{TM}}$, NeuroPace, Mountain View, CA). Responsive neurostimulation differs from other implantable stimulation devices like VNS and DBS because it is designed to deliver electrical stimulation in response to detected abnormal cortical electrical activity. The RNS system consists of one or two recording and stimulating depth or subdural cortical strip leads, which are connected to a programmable neurostimulator implanted in a craniectomy beneath the scalp. The ability to record cortical electrical activity is meaningful for the device because it allows for two distinct advantages:

- long-term, chronic ambulatory cortical recordings can be downloaded from the implanted RNS device, which may allow for a better understanding of a patient's seizure type, frequency, and onset location; and

- the device can be programmed to deliver stimulation when specified cortical electrical activity is detected, with the goal of reducing clinical seizure occurrence.

The RNS system's integrated detection-stimulation algorithms are part of a process termed 'closed-loop stimulation'. DBS and VNS, on the other hand, use 'open-loop stimulation' because the stimulation current is delivered according to a programmed pattern, independent of cortical activity.

Results of early RNS safety and efficacy trials were first reported in 2004 and 2009, 21,22 followed by recent publication of results from the RNS pivotal trial. The pivotal trial was a randomised, double-blind, sham-stimulation controlled study of stimulation in 191 adults with partial seizures, who had failed at least two AEDs prior to study enrollment. During the 12-week blinded evaluation period, those who were stimulated had a significantly greater mean seizure reduction (37.9\%) compared to the sham stimulation group (17.3\%). After the blinded evaluation period, the RNS device was turned 'on' in all study participants; long-term follow-up at one year demonstrated a responder rate ( $250 \%$ seizure reduction) of $46 \%$ of patients $(n=177)$ and at two years a responder rate of $46 \%(n=102)$. Safety endpoints from the trial were not higher than those reported in DBS implantation trials. Of note, $34 \%$ of trial patients had already undergone VNS stimulation prior to enrollment, and $32 \%$ had undergone prior therapeutic epilepsy surgery. Results were not stratified for these individuals, so the utility of RNS in patients who fail other surgical alternatives remains to be determined.

\section{Surgical Removal of Epileptogenic Tissue Temporal Lobectomy}

Resective surgery has long followed the principle of identifying and removing a focus of tissue responsible for seizure initiation (after confirming that the area is not responsible for a critical cortical function), or disconnecting areas that may be responsible for seizure propagation. Thus, resective surgery relies heavily on intensive pre-operative planning with advanced imaging and electrographic techniques in order to localise involved areas with a high degree of confidence. Present day advances in the realm of resective surgery are moving toward identifying pathological tissue and planning the 
optimal extent of resection, gathering long-term outcomes and integrating less invasive techniques such as stereotactic radiosurgery, radiofrequency ablation and MRI-guided laser produced thermal lesioning.

\section{Extent of Resection}

Poor prognosis in mesial temporal lobe epilepsy (MTLE) has been associated with hippocampal sclerosis as a histological finding, the signs of which are seen on MRI as hippocampal atrophy and T2 hyperintensity. ${ }^{23}$ As diagnostic imaging modalities have advanced significantly in recent years in terms of quality and resolution, imaging studies in epilepsy have shifted toward the quantification of temporal atrophy. Voxel-based morphometry (VBM) and pathological studies have demonstrated that tissue volume reduction in epilepsy patients extends beyond just the hippocampus to involve the entorhinal perirhinal, thalamic and temporopolar area.. ${ }^{24,25}$ These data lend credence to the idea that MTLE is a heterogeneous disease with variable tissue involved outside of just the hippocampus - a theory relevant to the long-standing debate surrounding the optimal extent of resection in MTLE surgery.

In the 1950s, Niemeyer introduced a limited resection by isolated removal of the mesial structures via a transcortical, transventricular selective amygdalohippocampectomy (SAH). ${ }^{26}$ As subsequent modifications in technique were described, ${ }^{27}$ including resection of the anterolateral temporal lobe, amygdala and hippocampus, there remained no clear evidence to support one particular method of resection over another. One of the strongest studies providing evidence that extent of hippocampal resection influences seizure outcome was a prospective randomised study by Wyler, published in 1995..$^{28}$ At one year, patients who had undergone total hippocampectomy (to the level of the superior colliculus) had $69 \%$ seizure-freedom, compared to $38 \%$ in those who had undergone partial hippocampectomy (to the anterior edge of the cerebral peduncle). In 2001, a randomised controlled trial established that temporal lobectomy $(6-6.5 \mathrm{~cm}$ of non-dominant or $4-4.5 \mathrm{~cm}$ of dominant anterior lateral temporal lobe) with amygdalohippocampectomy (at least 1-3 cm of anterior hippocampus) is more effective than medical therapy alone in patients with MRE. 29,30 Around the same time, a number of non-randomised studies reported outcomes based on variable resection of the anterolateral temporal lobe and mesial temporal structures, some of which suggested that larger extents of resection led to better outcomes. ${ }^{31-33}$ One prospective trial compared outcomes in patients who had received anterior temporal lobectomy (ATL) versus SAH and found no significant difference in seizure freedom at follow-up (72\% of ATL patients with mean follow up 6.7 years and $71 \%$ of SAH patients with mean follow up 4.5 years). ${ }^{34}$ However, small studies evaluating the volume of resected tissue based on analysis of post-operative MRI have suggested that patients who are seizure free have a larger volume of tissue resected than those who have persistent seizures, without having an effect on neuropsychological outcomes. ${ }^{35}$ More specifically, larger hippocampal resection, and more extensive amygdalohippocampal complex resection and total temporal resection have been associated with better outcomes. ${ }^{36,37}$

In order to address extent of mesial temporal resection in relation to outcome, results from a randomised trial of 2.5 versus $3.5 \mathrm{~cm}$ mesial temporal resection were recently described. ${ }^{38}$ Study patients received either partial temporal lobectomy or $\mathrm{SAH}$, and within these groups were randomised to 2.5 or $3.5 \mathrm{~cm}$ resection of the hippocampus-parahippocampal bloc. The authors found no significant difference in seizure freedom between all 2.5 and $3.5 \mathrm{~cm}$ resection groups (74 and $72.8 \%$, respectively). However, in subgroup analyses, the temporal lobectomy group had significantly higher seizure freedom compared to the SAH group (83.8 versus $67.2 \%$, $\mathrm{p}=0.013)$. The authors acknowledge the comparison of the temporal lobectomy group to the SAH group is subject to confounding factors and bias, since the trial was not designed to randomise patients between these two groups.

In sum, there is strong evidence that hippocampal resection should be at least $2.5 \mathrm{~cm}$, but no clear evidence that definitively favours one technique of resection over another in treating medically refractory MTLE, though a number of studies indicate that amygdalohippocampectomy along with a variable extent of anterolateral temporal resection achieves good seizure freedom outcomes.

\section{Long-term Outcomes}

In the wake of robust evidence that resective surgery for focal epilepsy carries a high likelihood of seizure remission, recent discussion has centred around the long-term durability of these effects. A meta-analysis of long-term outcomes for grouped temporal and extra-temporal surgery found a pooled seizure freedom rate of $62 \%$ for studies with five to 10 years' follow-up, but only $38 \%$ with more than ten years' follow-up. ${ }^{39}$ One group demonstrated that patients who underwent anterior temporal lobectomy, which across studies maintains higher seizure freedom rates than extra-temporal surgery, achieved only $41 \%$ seizure freedom at 10 years. ${ }^{40}$

A recent study examined long-term outcomes in a large cohort of 615 patients who underwent a variety of resective procedures for seizures, the pre-operative characteristics of which are unspecified. ${ }^{41}$ Amongst all patients who underwent a resective procedure, $47 \%$ were seizure free (or had simple partial seizures) at 10 years. Of those who underwent anterior temporal resection, $49 \%$ were seizure free at 10 years; temporal lesionectomy patients had the highest percentage seizure freedom at $56 \%$; those with extratemporal resections had a greater probability of seizure recurrence (31\% seizure free at 10 years). It therefore remains imperative in pre-operative planning to include a comprehensive discussion that seizures recur amongst certain populations in the long-term (>10 year) more than others, and may mandate continuation or implementation of pharmacological therapy.

\section{Stereotactic Radiosurgery}

The idea that MTLE could be treated with stereotactic radiosurgery (SRS) emerged as reports accumulated suggesting that stereotactic radiosurgery reduced seizure rates after lesional treatment (arteriovenous malformations, glial and metastatic tumours). ${ }^{42-44}$ SRS consists of precisely focused radiation delivered to an intracranial region of interest, selected using a fine-cut MRI and/or computer tomography (CT) scan. The MRI scan and radiation treatment are done with the patient in a stereotactic head-frame, which is fixed to the skull using percutaneous pins requiring only minimal local anaesthesia.

Despite its minimally invasive appeal, stereotactic radiosurgery presents unique concerns compared to open surgical resection because of two radiation-specific concepts: 
- tissue response to radiation, and thus the desired effects of radiosurgery, occurs in a delayed fashion compared to the immediate results of open surgical resection; and

- long-term follow-up is required to fully understand the deleterious effects of radiation on surrounding normal tissue, such as severe oedema and radiation-induced necrosis. Also, there is concern that there could be a significant risk of radiation-induced malignancy.

In 2004, results were published from the first prospective multicentre trial of SRS for 20 patients with drug-resistant MTLE. The radiation target included the anterior parahippocampal cortex, basal and lateral amygdala, and the hippocampus head and body. The authors reported a seizure freedom rate of $65 \%$ at two-year follow-up, with $45 \%$ experiencing visual field deficits and no observable neuropsychological deterioration. ${ }^{45}$ A subsequent multicentre prospective study randomised patients to high-dose (24 Gy) or low-dose (20 Gy) radiosurgery of the amygdala, hippocampus and parahippocampalgyrus. The authors reported $67 \%$ seizure freedom at three-year follow-up (76.9 \% in high dose and $58.8 \%$ in low dose); as anticipated, far fewer were seizure free at 12 months ( $30 \%)$, a time during which many patients experienced an exacerbation in their auras With regard to adverse events, $41 \%$ of low-dose and $61 \%$ of high-dose experienced visual field deficits, and overall $15 \%$ experienced verbal memory impairment. One patient experienced severe cerebral oedema with headaches and visual field deficits, ultimately requiring a temporal lobectomy. A recent follow-up report on neuropsychological outcomes demonstrated that cognitive outcomes, mood and quality of life had similar post-operative courses as seen in open surgery. ${ }^{46}$

Further data are necessary to determine the safety and efficacy of SRS in comparison with standard open surgical resection. A randomised, controlled trial - Radiosurgery or open surgery for epilepsy (ROSE) trial - is currently underway to compare gamma knife radiosurgery (GKRS) with open surgical temporal lobectomy for patients with medically refractory temporal lobe epilepsy. ${ }^{47}$

\section{Stereotactic Amygdalohippocampal Lesioning}

Stereotactic radiofrequency amygdalohippocampectomy (SAHE) was first described in $1978^{48}$ but has only recently emerged - with modern stereotactic techniques - as an alternative to open microsurgical resection. Radiofrequency amygdalohippocampectomy is performed under minimal sedation with local anaesthesia. The patient is placed in a stereotactic headframe and the trajectory planned using a fine-cut coronal MRI. A small percutaneous drill hole is made in the occipital entry area as defined by the pre-operative trajectory, and the electrode advanced through the hippocampal head to the amygdala. The thermocoagulation lesioning is then performed as the wire is withdrawn along the trajectory. ${ }^{49}$

Early outcomes from small patient series with one- to two-year follow-up have held promising results, with approximately $72-75 \%$ achieving seizure freedom (Engel Class I). ${ }^{50,51}$ Data at this point remain too preliminary, however, to draw concrete outcomes conclusions or compare radiofrequency lesioning with microsurgical resection. ${ }^{52} \mathrm{~A}$ newer technique for producing this hippocampal region lesioning has recently been developed. This involves stereotactic insertion of a catheter into the hippocampus and then using a laser to produce thermal lesioning while the patient is monitored in an MRI, to directly observe the tissue temperature and avoid lesioning outside of the desired target volume. Preliminary results appear promising, but as yet there is not adequate follow-up to permit analysis of this technique..$^{53}$

\section{Conclusions}

Epilepsy surgery has advanced to include a variety of stimulation, resective, and lesioning techniques that provide seizure reduction for patients with medically refractory epilepsy. With a greater breadth of surgical treatment options, perhaps it becomes paramount to ensure the multidisciplinary team has a comprehensive understanding of which treatment provides the highest likelihood of seizure remission while achieving collectively determined surgical goals. As the majority of novel surgical techniques have been employed for treating medically refractory partial seizures, it will be critical moving forward to gather long-term, prospective data regarding seizure freedom, reduction, and recurrence for each pathological subtype, in order to optimally tailor recommendations for each patient.
1. Zabara J, Inhibition of experimental seizures in canines by repetitive vagal stimulation, Epilepsia, 1992;33:1005-12.

2. Woodbury DM, Woodbury JW, Effects of vagal stimulation on experimentally induced seizures in rats, Epilepsia, 1990;31(Suppl. 2):S7-19.

3. Aalbers M, Vles J, Klinkenberg S, et al., Animal models for vagus nerve stimulation in epilepsy, Exp Neurol, 2011;230:167-75.

4. Dedeurwaerdere S, Vonck K, De Herdt V, et al. Neuromodulation with levetiracetam and vagus nerve stimulation in experimental animal models of epilepsy, Acta Neurol Belg, 2006;106:91-7.

5. Neu P, Heuser I, Bajbouj M, Cerebral blood flow during vagus nerve stimulation--a transcranial Doppler study, Neuropsychobiology, 2005:51:265-8.

6. Handforth A, DeGiorgio CM, Schachter SC, et al., Vagus nerve stimulation therapy for partial-onset seizures: a randomized active-control trial, Neurology, 1998;51:48-55.

7. Ben-Menachem $E$, Manon-Espaillat R, Ristanovic $R$, et al., Vagus nerve stimulation for treatment of partial seizures: 1. A controlled study of effect on seizures. First International Vagus Nerve Stimulation Study Group, Epilepsia, 1994;35:616-26.

8. Englot DJ, Chang EF, Auguste KI, Vagus nerve stimulation for epilepsy: a meta-analysis of efficacy and predictors of epilepsy: a meta-analysis of efficacy and pred
response, J Neurosurg, 2011:115(6):1248-55.

9. Follett KA, Weaver FM, Stern M, et al., Pallidal versus subthalamic deep-brain stimulation for Parkinson's disease, N Eng/J Med, 2010;362:2077-91.

10. Deuschl G, Schade-Brittinger $C$, Krack $P$, et al., A randomized trial of deep-brain stimulation for Parkinson's disease, N Engl J Med, 2006;355:896-908.
11. Lozano AM, Giacobbe P, Hamani C, et al., A multicenter pilot study of subcallosal cingulate area deep brain stimulation for treatment-resistant depression, I Neurosurg, 2012;116(2):315-22.

12. Goodman WK, Foote KD, Greenberg BD, et al., Deep brain stimulation for intractable obsessive compulsive disorder: pilot study using a blinded, staggered-onset design, Biol Psychiatry, 2010;67:535-42.

13. Ackermans $L$, Duits $A$, van der Linden $C$, et al., Double-blind clinical trial of thalamic stimulation in patients with Tourette syndrome, Brain, 2011;134:832-44.

14. Wright $\mathrm{GD}$, MCLellan $\mathrm{DL}$, Brice JG, A double-blind trial of chronic cerebellar stimulation in twelve patients with severe epilepsy, J Neurol Neurosurg Psychiatry, 1984:47:769-74.

15. Gigante PR, Goodman RR, Alternative surgical approaches in epilepsy, Curr Neurol Neurosci Rep, 2011:11:404-8.

16. Velasco M, Velasco F, Velasco AL, et al., Subacute electrical stimulation of the hippocampus blocks intractable temporal lobe seizures and paroxysmal EEG activities, Epilepsia, 2000;41:158-69.

17. Fisher R, Salanova V, Witt T, et al., Electrical stimulation of the anterior nucleus of thalamus for treatment of refractory epilepsy, Epilepsia, 2010:51:899-908.

18. US Food and Drug Administration, Neurological Devices Panel Meeting Available at: www fda.gov/downloads/ AdvisoryCommittees/CommitteesMeetingMaterials/MedicalDe AdvisoryCommittees/Committees/MeetingMaterials/MedicalDe
vices/MedicalDevicesAdvisoryCommittee/NeurologicalDevices Panel/UCM206524.pdf (accessed 15 November 2011).

19. Health USNIO, Controlled Randomized Stimulation Versus Resection (CoRaStiR). Available at: http://clinicaltrials.gov/ ct2/show/NCT00431457 (accessed 15 November 2011).

20. Health USNIo, A Multicenter Study of hippocampal electrical stimulation (METTLE) 2011. Available at: http://clinicaltrials.gov/ct2/show/NCT00717431?term= A+Multicenter+Study+of+Hippocampal+Electrical+ Stimulation.\&rank=1 (accessed 15 November 2011).

21. Morrell MJ, RNS System Pivotal Investigators. Results of a multicenter double blinded randomized controlled pivotal investigation of the RNS(TM) system for treatment of intractable partial epilepsy in adults, Presented at: the 63rd Annual American Epilepsy Society Meeting, Boston, MA, US 4-8 December 2009

22. Vossler D, Doherty M, Goodman R, et al., Early safety experience with a fully implanted intracranial responsive neurostimulator for epilepsy, Presented at: the 58th Annua Meeting of the American Epilepsy Society Meeting, New Orleans, Louisiana, US, 3-7 December 2004

23. Henry TR, Chupin M, Lehericy S, et al., Hippocampal sclerosis in temporal lobe epilepsy: findings at $7 \mathrm{~T}(1)$, Radiology, 2011;261:199-209

24. Labate A, Cerasa A, Aguglia U, et al., Voxel-based morphometry of sporadic epileptic patients with mesiotemporal sclerosis, Epilepsia, 2010;51:506-10

25. Bernasconi N, Duchesne S, Janke A, et al., Whole-brain voxel-based statistical analysis of gray matter and white matter in temporal lobe epilepsy, Neuroimage, 2004;23:717-23.

26. Niemeyer $\mathrm{P}$, The transventricular amygdalohippocampectomy in temporal lobe epilepsy, Springfield, Illinois: CC Thomas, 1958.

27. Yasargil MG, Teddy PJ, Roth P, Selective amygdalohippocampectomy. Operative anatomy and surgica technique, Adv Tech Stand Neurosurg, 1985;12:93-123.

28. Wyler AR, Hermann BP, Somes G, Extent of medial temporal resection on outcome from anterior temporal lobectomy: a randomized prospective study, Neurosurgery, 1995;37:982-90. 
29. Wiebe S, Blume WT, Girvin JP, et al., A randomized, controlled trial of surgery for temporal-lobe epilepsy, N Engl I Med, 2001:345:311-8.

30. Spencer SS, Berg AT, Vickrey BG, et al., Initial outcomes in the Multicenter Study of Epilepsy Surgery, Neurology, 2003;61:1680-5.

31. Goldring S, Edwards I, Harding GW, et al., Results of anterio temporal lobectomy that spares the amygdala in patients with complex partial seizures, I Neurosurg, 1992;77:185-93.

32. Feindel $W$, Rasmussen $T$, Temporal lobectomy with amygdalectomy and minimal hippocampal resection: review of 100 cases, Can I Neurol Sci, 1991;18:603-5.

33. Olivier A, Relevance of removal of limbic structures in surgery for temporal lobe epilepsy, Can I Neurol Sci, 1991;18:628-35

34. Paglioli E, Palmini A, Portuguez $M$, et al., Seizure and memory outcome following temporal lobe surgery: selective compared with nonselective approaches for hippocampal sclerosis, I Neurosurg, 2006;104:70-8.

35. Shamim S, Wiggs E, Heiss J, et al., Temporal lobectomy: resection volume, neuropsychological effects, and seizure outcome, Epilepsy Behav, 2009;16:311-4.

36. Awad IA, Katz A, Hahn JF, et al., Extent of resection in temporal lobectomy for epilepsy. I. Interobserver analysis and correlation with seizure outcome, Epilepsia, 1989;30:756-62.

37. Joo EY, Han HJ, Lee EK, et al., Resection extent versus postoperative outcomes of seizure and memory in mesial temporal lobe epilepsy, Seizure, 2005;14:541-51.
38. Schramm J, Lehmann TN, Zentner J, et al., Randomized controlled trial of $2.5-\mathrm{cm}$ versus $3.5-\mathrm{cm}$ mesial temporal rontion in temporal lobe epis 3.5-cme part 1: intent-to-treat resection in tem

39. Tellez-Zenteno JF, Dhar R, Wiebe $\mathrm{S}$, Long-term seizure outcomes following epilepsy surgery: a systematic review and meta-analysis, Brain, 2005;128:1188-98

40. McIntosh AM, Kalnins RM, Mitchell LA, et al., Tempora lobectomy: long-term seizure outcome, late recurrence and risks for seizure recurrence, Brain, 2004;127:2018-30.

41. de Tisi J, Bell GS, Peacock JL, et al., The long-term outcome of adult epilepsy surgery, patterns of seizure remission, and relapse: a cohort study, Lancet, 2011;378:1388-95.

42. Heikkinen ER, Konnov B, Melnikov L, et al., Relief of epilepsy by radiosurgery of cerebral arteriovenous malformations, Stereotact Funct Neurosurg, 1989:53:157-66.

43. Kida Y, Kobayashi T, Tanaka T, et al., Seizure control afte radiosurgery on cerebral arteriovenous malformations, I Clin Neurosci, 2000;7(Suppl. 1):6-9.

44. Schrottner $\mathrm{O}$, Eder HG, Unger $\mathrm{F}$, et al., Radiosurgery in lesional epilepsy: brain tumors, Stereotact Funct Neurosurg, 1998;70(Suppl. 1):50-6

45. Regis J, Rey M, Bartolomei F, et al., Gamma knife surgery in mesial temporal lobe epilepsy: a prospective multicenter mesial temporal lobe epilepsy.

46. Quigg M, Broshek DK, Barbaro NM, et al., Neuropsychological outcomes after Gamma Knife radiosurgery for mesial temporal lobe epilepsy: a prospective multicenter study,
Epilepsia, 2011;52:909-16

47. Health UNIo, Radiosurgery or Open Surgery for Epilepsy Trial (ROSE). Available at: http://clinicaltrials.gov/ct2/show/ NCT00860145?term=radiosurgery+and+surgery+ epilepsy\&rank=1 (accessed 2 December 2011)

48. Vladyka V, [Surgical treatment of epilepsy and its application in temporal epilepsy], Cesk Neurol Neurochir, 1978;41:95-106.

49. Liscak R, Malikova H, Kalina M, et al. Stereotactic radiofrequency amygdalohippocampectomy in the treatment of mesial temporal lobe epilepsy, Acta Neurochir (Wien), 2010;152:1291-8.

50. Kalina M, Lisck R, Vojtech Z, et al., Stereotactic amygdalohippocampectomy for temporal lobe epilepsy: promising results in 16 patients, Epileptic Disord 2007:9(Suppl. 1):S68-74.

51. Malikova H, Vojtech Z, Liscak R, et al., Stereotactic radiofrequency amygdalohippocampectomy for the treatment of mesial temporal lobe epilepsy: correlation of MRI with clinical seizure outcome, Epilepsy Res, 2009;83:235-42.

52. Malikova H, Vojtech Z, Liscak R, et al., Microsurgical and stereotactic radiofrequency amygdalohippocampectomy for the treatment of mesial temporal lobe epilepsy: different volume reduction, similar clinical seizure control, Stereotact Funct Neurosurg, 2010;88:42-50.

53. Curry D, Gowda A, MCNichols R, et al., Stereotactic MRI-guided Laser Ablation of Epileptogenic Foci in Children Presented at: the American Epilepsy Society 65th Annua Meeting, Baltimore, MD, US, 2-6 December 2011. 